\title{
Comportamiento de mullitas a alta temperatura: Estudio mediante Difracción de Rayos X
}

\author{
M.P. VILLAR' ${ }^{1}$, L. GAGO-DUPORT², R. GARCÍA ${ }^{1}$ \\ 'Dpto. Ciencia de los Materiales e Ingeniería Metalúrgica y Química Inorgánica. Universidad de Cádiz. 11510-Puerto Real (Cádiz) \\ ${ }^{2}$ Dpto. Geociencias Marinas y Ordenación del Territorio. Universidad de Vigo. 36200-Vigo (Pontevedra)
}

\begin{abstract}
En este trabajo se exponen los resultados obtenidos del seguimiento, mediante difracción de rayos $\mathrm{X}$ en cámara de alta temperatura, del comportamiento frente a la dilatación térmica de diversas mullitas $\left(3 \mathrm{Al}_{2} \mathrm{O}_{3} \cdot 2 \mathrm{SiO}_{2}\right)$ sol-gel dopadas con contenidos del 1, 3, 6 y $9 \%$ en peso de $\mathrm{Cr}_{2} \mathrm{O}_{3}$. La presencia de cromo permite obtener materiales con ausencia de fase vítrea y microestructuras homogéneas, lo cual resulta de enorme interés ya que la presencia de vidrio en estos materiales supone una degradación de sus propiedades mecánicas, sobre todo en regímenes de funcionamiento a alta temperatura. Los resultados de dicho estudio revelan que la introducción de cromo en la red de mullita produce una reducción efectiva de su coeficiente lineal de expansión térmica, aunque también un aumento de la anisotropía con que dicha expansión tiene lugar. Únicamente con dopados de un $6 \%$ en peso de $\mathrm{Cr}_{2} \mathrm{O}_{3}$ se consiguen comportamientos similares al de la mullita no dopada en cuanto a anisotropía en la dilatación de la red.
\end{abstract}

Palabras clave: Expansión térmica, mullita, difracción de rayos X.

\section{High-temperature behaviour of mullites: Study by means of X-Ray Diffraction}

Results on thermal behaviour of several sol-gel chromium doped mullites $\left(3 \mathrm{Al}_{2} \mathrm{O}_{3} \cdot 2 \mathrm{SiO}_{2}\right.$ with $\mathrm{Cr}$ contents of $1,3,6$ and 9 wt.\% $\left.\mathrm{Cr} \mathrm{O}_{3}\right)$ by means of high-temperature $X$-ray diffraction are exposed in this work. Chromium additions make possible to obtain glass-free materials with very homogeneous microstructures, which is highly convenient as the presence of glass in these materials provides a certain degradation on their mechanical properties, especially at high temperature operations. This study reveals that the chromium introduction in the mullite lattice promotes an effective reduction of its linear thermal expansion coefficient, although also an increase in the anisotropy with which this expansion takes place. Only with the $6 \mathrm{wt} . \% \mathrm{Cr}_{2} \mathrm{O}_{3}$ content the anisotropy for the lattice expansion is similar to the non-doped mullite.

Key words: Thermal expansion, mullite, X-ray diffraction.

\section{INTRODUCCIÓN}

La mullita, aluminosilicato de estequiometría $\mathrm{Al}_{4+2 x} \mathrm{Si}_{2-2 x} \mathrm{O}_{10-x}$ $(0.15<\mathrm{x}<0.60$, donde $x$ indica el número de vacantes de oxígeno por celda unidad) es el componente mayoritario de muchas cerámicas tradicionales. Además, la Ingeniería de Cerámicas moderna la ha situado en un lugar de privilegio en cuanto a aplicaciones técnicas de alta temperatura se refiere. Las propiedades que la han hecho merecer esta consideración son, entre otras, su baja expansión térmica, así como su excelente resistencia al choque térmico, en conjunción con una gran resistencia a la fluencia (1). Estas propiedades, además de la baja constante dieléctrica y baja conductividad térmica de la mullita, permiten a la tecnología SOC (silicon-on-ceramics) plantear la utilización de estas cerámicas mullita como sustrato de diversos dispositivos. Diversos autores han conseguido materiales de mullita con coeficientes de expansión térmica bien ajustados a los del silicio $(2,3)$. Sin embargo, los contenidos de vidrio en dichos materiales son apreciables, y ello supone una degradación más o menos importante de sus propiedades mecánicas a altas temperaturas. Una alternativa consiste en la adición de cromo, que permite obtener materiales libres de fase vítrea en límites de grano (4).

En este contexto, el estudio del comportamiento de estos materiales con composiciones incluidas en el sistema $\mathrm{Al}_{2} \mathrm{O}_{3}-\mathrm{SiO}_{2}-\mathrm{Cr}_{2} \mathrm{O}_{3}$ (5) cuando son sometidos a condiciones de operación de altas temperaturas resulta fundamental. Los experimentos de difracción constituyen una herramienta adecuada para realizar dicho estudio, tal y como avalan publicaciones recientes referidas a diferentes mullitas $(6,7)$. Trabajos anteriores también recogen medidas de expansión térmica para materiales de mullita $(8,9)$, aunque con la limitación de disponer de datos basados en calentamientos hasta $900^{\circ} \mathrm{C}$.

\section{PARTE EXPERIMENTAL}

Los materiales utilizados en este estudio han sido mullitas obtenidas mediante sol-gel (10) y sinterizadas a $1650^{\circ} \mathrm{C}$, con 5 composiciones diferentes: mullita $3: 2$ y con adiciones de $1,3,6$ y $9 \%$ en peso de $\mathrm{Cr}_{2} \mathrm{O}_{3}$. Los experimentos de difracción de rayos $\mathrm{X}$ se realizaron sobre piezas pulidas de dimensiones $3 \times 4 \times 1.5 \mathrm{~mm}$. Dichos experimentos se llevaron a cabo en un difractómetro Philips PW-1830 ( $\lambda=0.15406 \mathrm{~nm})$ equipado con una cámara de alta temperatura H.T.K. 10 de Anton-Peer, desde temperatura ambiente hasta $1590^{\circ} \mathrm{C}$ (mullita no dopada y dopada con $1 \%$ en peso de $\mathrm{Cr}_{2} \mathrm{O}_{3}$ ) y $1469^{\circ} \mathrm{C}$ (resto de las muestras), con incrementos sucesivos de temperatura de $66^{\circ} \mathrm{C}$. El rango de colección de datos fue de $10-80^{\circ} 2 \theta$, con intervalos de $0.02^{\circ}$, y tiempo de adquisición de $1 \mathrm{~s} /$ paso.

La determinación de los parámetros de red se realizó siguiendo una rutina de ajuste de los difractogramas utilizando el programa FULLPROF (11). El modelo estructural de partida contenía las fases mullita y $\mathrm{Al}_{2} \mathrm{O}_{3}-\mathrm{Cr}_{2} \mathrm{O}_{3}$ cuando esta última se detectaba en los materiales dopados $(4,5)$. Los factores de acuerdo obtenidos en los refinamientos fueron $\mathrm{R}_{\mathrm{wp}}=7-11 \%, \mathrm{R}_{\mathrm{p}}=6-8 \%$ y Gof $=1.2-1.6$, llevando el factor $\mathrm{R}_{\mathrm{Bragg}}$ a valores de 0.5 .

\section{RESULTADOS Y DISCUSIÓN}

La Figura 1a-c presenta la dependencia de los parámetros de red de la mullita con la temperatura para los diferentes materiales sometidos a estudio. Un hecho destacable en todos los materiales es una inflexión de las curvas correspondientes a los parámetros reticulares $a$ 
y $\boldsymbol{b}$ en el intervalo de temperaturas $700-900^{\circ} \mathrm{C}$ (Figura 1a,b), de manera que, por debajo de esta intervalo, todas las curvas son prácticamente lineales y paralelas (con desviaciones de la linealidad a valores bajos de temperatura, $17-300^{\circ} \mathrm{C}$, aproximadamente) mientras que, por encima, se definen dos comportamientos diferentes de las mullitas en función de su composición: confluencia de las curvas correspondientes a la mullita no dopada y la que contiene un $1 \%$ en peso de $\mathrm{Cr}_{2} \mathrm{O}_{3}$, por una parte, y de las curvas de las mullitas dopadas con 6 y $9 \%$ en peso de $\mathrm{Cr}_{2} \mathrm{O}_{3^{\prime}}$ por otra. Este hecho sugiere un esquema de competición entre los desplazamientos térmicos anisotrópicos que afectan a las interacciones metal-ion y la dilatación inicial inducida por el porcentaje de $\mathrm{Cr}^{3+}$ que sustituye al $\mathrm{Al}^{3+}$ en los octaedros de la estructura, de modo que a temperaturas elevadas (superiores a $800^{\circ} \mathrm{C}$, aproximadamente) prima el efecto de los desplazamientos térmicos de los átomos. En este sentido, el porcentaje de $3 \%$ de peso en $\mathrm{Cr}_{2} \mathrm{O}_{3}$ resulta crítico, puesto que para contenidos de dopantes superiores no aumentaría la dilatación térmica en consonancia con la cantidad de dopante, y la razón podría ser que las diferencias en los radios iónicos se minimizarían por las largas amplitudes de vibración de los parámetros de Debye-Waller en la dirección de los ejes $\boldsymbol{a}$ y $\boldsymbol{b}$ de la celda. De este modo quedaría explicado el comportamiento final observado, a temperaturas cercanas a los $1500^{\circ} \mathrm{C}$, con los últimos puntos de las curvas convergiendo con independencia del contenido de $\mathrm{Cr}$, especialmente en la constante reticular $\boldsymbol{b}$ (Figura 1b). El parámetro $\boldsymbol{c}$, en cambio, sigue una evolución con la temperatura completamente diferente (Figura 1c). Aunque de forma menos significativa, este intervalo de temperatura también incide en la evolución del eje $c$ frente a la dilatación térmica aunque, en este caso, las amplitudes de vibración en esta dirección no son tan amplias como para superar el efecto de expansión de la red debida al mayor tamaño del catión $\mathrm{Cr}^{3+}$ (12). Son las mullitas de mayor contenido de dopante las que presentan mayor desviación de la linealidad de la expansión a partir de esta temperatura, lo cual sería indicativo de que a elevados contenidos de $\mathrm{Cr}_{2} \mathrm{O}_{3}$ se favorece la incorporación de éste formando agregados en los canales estructurales paralelos al eje $c$ de entorno octaédrico u octaédrico distorsionado.

La introducción de cromo en la red de mullita produce, por otra parte, un continuo decremento de sus coeficientes lineales de expansión térmica (Tabla I), aunque también un aumento progresivo del factor de anisotropía (éste se anula para materiales que sufren expansión isotrópica). Únicamente la muestra con un $6 \%$ en peso de $\mathrm{Cr}_{2} \mathrm{O}_{3}$ presenta un factor de anisotropía similar al de la mullita no dopada.

TABLA I. COEFICIENTES LINEALES DE EXPANSIÓN TÉRMICA Y FACTORES DE ANISOTROPÍA ENTRE $200 \mathrm{y} 1469^{\circ} \mathrm{C}$.

\begin{tabular}{|c|c|c|c|c|c|}
\hline & \multicolumn{4}{|c|}{ Coeficientes lineales de expansión térmica $\left(x 10^{-6 /} /{ }^{\circ} \mathrm{C}\right)$} & \multirow[b]{2}{*}{$A^{b}$} \\
\hline Material & $\alpha(a)$ & $\alpha(b)$ & $\alpha(c)$ & $\alpha(V)^{a}$ & \\
\hline No dopado & 4.13 & 6.83 & 6.09 & 5.68 & 5.40 \\
\hline $1 \% \mathrm{Cr}_{2} \mathrm{O}_{3}$ & 3.19 & 6.30 & 6.41 & 5.30 & 6.44 \\
\hline $3 \% \mathrm{Cr}_{2} \mathrm{O}_{3}$ & 2.35 & 5.71 & 5.16 & 4.41 & 6.72 \\
\hline $6 \% \mathrm{Cr}_{2} \mathrm{O}_{3}$ & 2.57 & 4.95 & 5.37 & 4.30 & 5.60 \\
\hline $9 \% \mathrm{Cr}_{2} \mathrm{O}_{3}$ & 1.88 & 4.11 & 5.55 & 3.85 & 7.34 \\
\hline
\end{tabular}

Coeficiente lineal de expansión volumétrico medio, dado por $\alpha(V)=[\alpha(a)+\alpha(b)+\alpha(c)] / 3$ ${ }^{b}$ Factor de anisotropía, $\mathrm{A}=(|\alpha(b)-\alpha(c)|+|\alpha(b)-\alpha(a)|+|\alpha(c)-\alpha(a)|) \cdot\left(10^{-6}\right)$

\section{CONCLUSIONES}

Este estudio pone de manifiesto que la introducción de cromo en mullita conduce a una disminución efectiva del coeficiente de expansión térmica de las cerámicas dopadas, aunque dicha expansión se hace más anisotrópica. Al mismo tiempo, han quedado establecidas las pautas de comportamiento de la red de mullita con la temperatura en función de su composición, observando diferencias en la dilatación sufrida por los distintos ejes de la celda. El contenido de dopante del $3 \%$ en peso de $\mathrm{Cr}_{2} \mathrm{O}_{3}$ resulta definitivo al marcar diferencias de comportamiento frente a la dilatación a temperaturas superiores a $800^{\circ} \mathrm{C}$. Los valores de los parámetros reticulares $\boldsymbol{a}$ y $\boldsymbol{b}$ tienden a confluir a la temperatura más elevada.
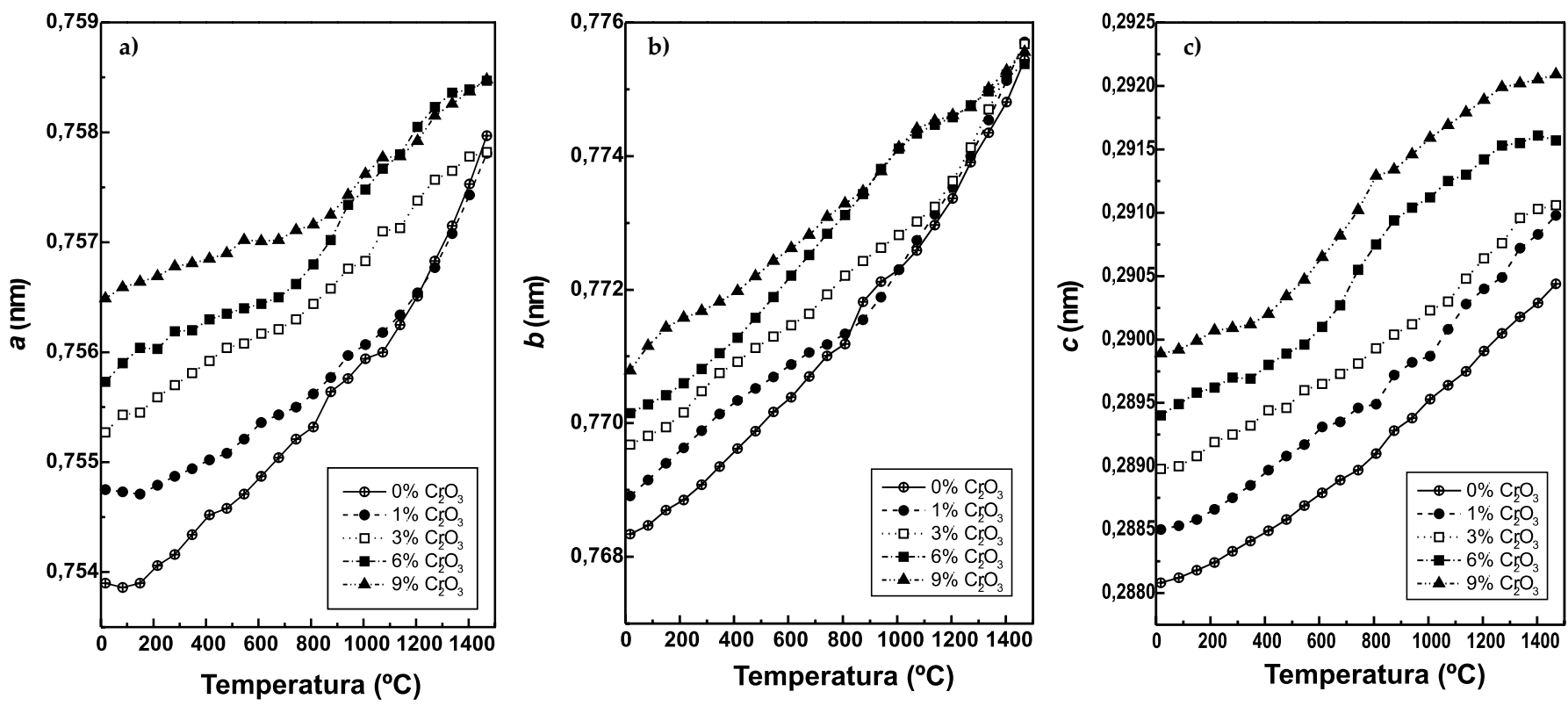

Figura 1. Evolución de los parámetros reticulares $\boldsymbol{a}, \boldsymbol{b}$ y $\boldsymbol{c}$ de la mullita 3:2 y de las mullitas dopadas con cromo frente a la temperatura 


\section{AGRADECIMIENTOS}

Las medidas de difracción se realizaron en la División de Difracción de Rayos X de los Servicios Centrales de Ciencia y Tecnología, en la Universidad de Cádiz. Los autores agradecen a los Dres. Saruhan y Schneider (DLR, Colonia, Alemania) el suministro de los materiales sinterizados. Uno de los autores, LGD, agradece al MCyT el apoyo económico recibido a través del proyecto BTE-2000-0877. MPV y RG agradecen la financiación de la Junta de Andalucía (Grupo de Investigación del PAI TEP-0120).

\section{BIBLIOGRAFÍA}

1. H. Schneider, K. Okada, J.A. Pask. Mullite and Mullite Ceramics. J. Wiley \& Sons, UK, 1994.

2. J. Requena, J.F. Bartolomé, J.S. Moya, S. de Aza, F. Guitián, G. Thomas. «Mullite-aluminosilicate Glassy Matrix Substrates Obtained by Reactive Coating». J. Eur. Ceram. Soc. 16 [2] 249-254 (1996).

3. M. Ohashi, Y. Ilida, S. Wada. «Mullite-based Substrates for Polycrystalline Silicon Thin-film Solar Cells». J. Ceram. Soc. Japan 108 [1] 105-107 (2000).
4. M.P. Villar. «Cristalización y desarrollo microestructural de mullitas sol-gel dopadas con cromo». Tesis Doctoral. Universidad de Cádiz (2000).

5. P.L. Roedor, F.P. Glasser, E.F. Osborn. «The system $\mathrm{Al}_{2} \mathrm{O}_{3}-\mathrm{Cr}_{2} \mathrm{O}_{3}-\mathrm{SiO}_{2}$ » J. Am. Ceram. Soc. 51 [10] 585-594 (1968).

6. W.M. Kriven, J.W. Palko, S. Sinogeikin, J.D. Bass, A. Sayir, G. Brunauer, H. Boysen, F. Frey, J. Schneider. «High temperature single crystal properties of mullite». J. Eur. Ceram. Soc. 19 [13-14] 2529-2541 (1999).

7. G. Brunauer, F. Frey, H. Boysen, H. Schneider. «high temperature thermal expansion of mullite: an in situ neutron diffraction study up to $1600^{\circ} \mathrm{C}$. J. Eur. Ceram. Soc. 21 [14] 2563-2567 (2001).

8. H. Schneider, E. Eberhard. «Thermal expansion of mullite». J. Am. Ceram. Soc. 73 [7] 2073-2076 (1990).

9. H. Schneider, K. Rodewald, E. Eberhard. «Thermal expansion discontinuities of Mullite». J. Am. Ceram. Soc. 76 [11] 2896-2898 (1993).

10. H. Schneider, K. Ikeda, B. Saruhan, H. Rager. «Electron paramagnetic resonance and optical absorption studies on Cr-doped mullite precursors». J. Eur. Ceram. Soc. 16 [2] 211-215 (1996).

11. J. Rodríguez-Carvajal. «Short Reference Guide of the Program FULLPROF, Version 3.5». La. Leon Brouillon (1995).

12. M.P. Villar, J.M. Geraldía y L. Gago-Duport. «Structural disorder and thermal dilation behavior in Cr-doped mullite». Mat. Res. Soc. Symp. Proc. 136 757-762 (1994)

Recibido: 1.2 .03

Aceptado: 30.11 .03 